\title{
Diversidad y abundancia ictiofaunística del río Grande de Térraba, sur de Costa Rica
}

\author{
José Rodrigo Rojas M. ${ }^{1}$ \& Omar Rodríguez S. ${ }^{2}$ \\ 1. Proceso de Planeamiento Ambiental, Centro Nacional de Planificación Eléctrica, Instituto Costarricense de \\ Electricidad, Apdo 10032-1000, San José. Costa Rica; rrojasm@ice.go.cr; yoyi66@yahoo.com, \\ 2. Biólogo Investigador; omarrodriguez@yahoo.com.mx
}

\author{
Recibido 19-VII-2007. Corregido 02-V-2008. Aceptado 26-V-2008.
}

\begin{abstract}
The diversity, abundance and distributional pattern of freshwater fish communities in the Térraba River, south Costa Rica, were investigated from the early dry season of 2004 to early rain season of 2005 . There have been no preview studies on the freshwater fish distribution in Térraba. Therefore, the aims of this study were to determine fish species richness, abundance and distribution there. Fish sampling was done using a combination of gears such as gill net, fine mesh net and visual observation. Thirty three species, 26 genera and 14 families were collected in four sampling sites along the river. The number and biomass captured during the entire study was 984 individuals and $147410.9 \mathrm{~g}$ respectively. Most of them are carnivorous species (48\%), $33.3 \%$ are omnivorous and $12 \%$ detritivorous, and only two species are herbivorous. The most important species in relative abundance $(56.5 \%)$ and biomass $(53.7 \%)$ in the study area was the machaca (Brycon behreae). It should be clear that although the list of fish species that occur in Térraba River is reasonably complete, knowledge of their ichthyogeographic history patterns is superficial. The main community component was secondary freshwater species; with 17 invading brackish water species and one introduced species (tilapia O. niloticus). Nine species are reported for the first time in this river. The diversity index $\mathrm{H}^{\top}$ varied from 2.32 (E1 Brujo) to 1.67 (Coto), a similar pattern was also showed for the other indexes. Most of our results were similar to those of previous studies on freshwater fish distribution elsewhere, however no significant correlation between species distribution and environmental variables was found, and we hypothesized that the depth and water velocity and geomorphological are major environmental variables that influence the fish distribution. Our findings are opposed to the tendency, for species composition, to increase from upstream to the mouth of the river, which is probably due to two major human activities (discharge of waste of pineapple fields and sediments in the main channel). These activities could constitute in the future a real threat for the fish population and other aquatic organisms. Growth rates, competition, predation pressures, community organization, biotic versus physical factors in relation to distribution, and many other aspects of fish biology remain to be studied. Future surveys will involve more quantitative surveys in different seasons and over time to monitor the long-term variation in the diversity and abundance of freshwater fish species in Térraba. Rev. Biol. Trop. 56 (3): 1429-1447. Epub 2008 September 30 .
\end{abstract}

Key words: freshwater fishes, species richness, abundance, spatial and temporal distribution, River Grande de Térraba, Costa Rica.

Costa Rica, y en especial en la vertiente pacífica, ha experimentado en las últimas décadas un incremento en los estudios sobre los factores ecológicos que determinan la distribución y estructura comunitaria de los peces (Bussing 1974a, b, 1976, Bussing y López 1977, Constantz et al. 1981, Winemiller 1983, Alpírez 1985, Burcham 1988, Winemiller y Morales 1989, Lyons y Schneider 1990, Wootton y Oemke 1992, Winemiller 1993, Bussing et al. 1994, Rojas et al. 1994, Chicas 2001), no obstante resultan insuficientes si consideramos la velocidad del deterioro e impactos ambientales negativos a los que están expuestas la mayoría de los ríos de esta vertiente (Estado de la Nación 2006). El río Grande de Térraba 
no es la excepción, ya que aunque las referencias sobre este ecosistema se remontan a épocas precolombinas (Linares y Ranere 1980, Corrales et al. 1988), la conquista (Camacho 1967) y al legado hecho por diversos ictiólogos (Regan 1907, 1908, Meek 1914, Briggs 1984, Bussing 1966, 1974 a, b, 1980, 1985a, b) prevalece como un ecosistema ecológicamente desconocido y con una clara degradación y pérdida de calidad ambiental. En ese sentido Rojas y Rodríguez (2005 y 2006) señalan aspectos puntuales sobre la diversidad de peces y su importancia en la gestión ambiental de obras de generación hidroeléctrica, sin embargo aún los vacíos son evidentes.

Ictiológicamente el río Grande de Térraba pertenece a la Provincia Ístmica (Bussing 1998) y es uno de los ecosistemas riverinos más importantes del Pacífico Sur de Costa Rica, no por la compleja red acuática que reúne, sino porque desemboca en la zona deltáica del Humedal Nacional de Térraba-Sierpe, área protegida con la mayor superficie de bosque de manglar de Costa Rica y uno de los más grandes de Centro América (UICN-ORMA 1995). Este río, de acuerdo con Vannote et al. (1980) y Honran et al. (2000), podría ser un buen ejemplo de biotopo con comunidades hidrobiológicas con fluctuaciones y gradientes ecológicos que influyen en la composición, distribución y estructura de las comunidades de peces y cuya complejidad aumenta conforme disminuye en altitud. La dinámica de adición y sustitución de especies, que mencionó Schlosser (1991), podría acentuarse en el Térraba debido a las discontinuidades geomorfológicas y/o condiciones abióticas. El río podría comportarse como los ecosistemas templados y/o tropicales donde la diversidad íctica depende de la dinámica de recolonización y de la habilidad de los peces para encontrar refugios apropiados (Meffe y Minkley 1987 y Menge y Olson 1990). Es previsible que en el tramo inferior donde las aguas fluyen a baja velocidad, con alta sedimentación, menor concentración de oxígeno, orillas con pastizales y zacatales y sustrato arenosolodoso, aumente la complejidad estructural y funcional expresada en términos de una mayor riqueza y diversidad ictiofaunística. Además es posible que se trate de una zona de transición entre ictiofauna estrictamente de agua dulce con especies estuario-marinas. Evidentemente, no existe una serie de especulaciones sobre la dinámica ecológica del río Grande de Térraba, sino que también hay hipótesis sobre las afectaciones que experimentaría ante la construcción de infraestructura, por tanto se ha planteado una investigación cuyo primera fase tiene como propósito analizar, describir y comparar la presencia, distribución, abundancia y patrones de diversidad de la ictiofauna, como una herramienta efectiva de gestión ambiental que permita generar escenarios de impacto así como estrategias de mitigación.

\section{MATERIAL Y MÉTODOS}

Descripción del área de estudio: Las estaciones de muestreo se ubican en la cuenca del río Grande de Térraba, en el sector sur de la vertiente del Pacífico $\left(9^{\circ} 35^{\prime} 11^{\prime}{ }^{\prime}-8^{\circ} 45^{\prime} 01\right.$ ' $\mathrm{N}$, $82^{\circ} 40$ '22"- 8350'11'”W), cantón de Osa, Provincia de Puntarenas (Fig. 1). La cuenca hidrográfica mide $5.200 \mathrm{~km}^{2}$ y representa el $10 \%$ del territorio nacional. Está constituida por los ríos Chirripó, Buena Vista, General, Ceibo, Cabagra, Cotón y Coto Brus. El patrón climático corresponde a un régimen típico del Pacífico, influido en septiembre y octubre, por los huracanes y tormentas tropicales del Caribe. Las lluvias máximas se registra en octubre y las mínimas en febrero-marzo. Los valores de precipitación promedio son alrededor de 3300 $\mathrm{mm}$, disminuyendo hacia las partes bajas de la zona costera, donde alcanza valores cercanos a los de $2000 \mathrm{~mm}$. La humedad relativa anual es mayor del $80 \%$, febrero y marzo son menor humedad y octubre y noviembre los de mayor (ICE 1999).

Trabajo de campo y análisis de datos: Previo al inicio del trabajo de campo se navegó la zona de estudio y mediante buceos con "snorkel" y mascarilla se seleccionaron los sitios de muestreo tratando de incluir la complejidad estructural típica de un río tropical, es 


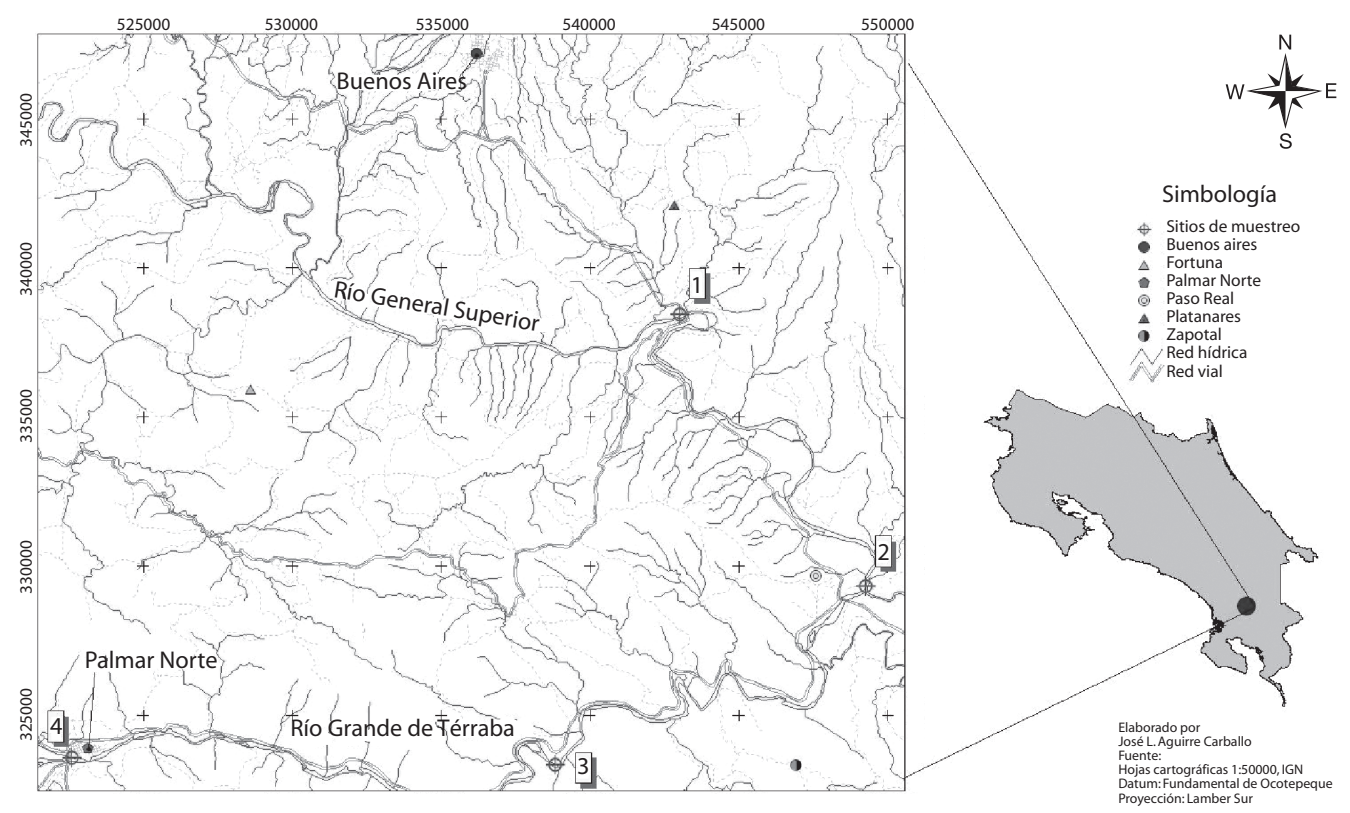

Fig. 1. Mapa del área de estudio, río Grande de Térraba, Costa Rica.

Fig. 1. Map of study, Térraba river, Costa Rica.

decir zonas de pozas, sectores con rápidos, fondos arenosos, lodosos, pedregosos, así como estaciones con vegetación en sus alrededores. Entre febrero 2004 y abril del 2005 se llevaron a cabo 14 muestreos en el tramo estudiado, el cuál se dividió en cuatro estaciones (1- Puente sector El Brujo a 145 msnm, 2- confluencia del río Coto Brus con el río General a $95 \mathrm{msnm}$, 3- poza Lagarto a $55 \mathrm{msnm}$ y 4- puente Palmar a $15 \mathrm{msnm}$ ). Entre cada estación hay aproximadamente $18 \mathrm{~km}$ y entre la estación uno y cuatro hay $55 \mathrm{~km}$.

La captura y liberación de la ictiofauna se hizo de día utilizando una red agallera de $25 \mathrm{x}$ $1.80 \mathrm{~m}$ y $8.9 \mathrm{~cm}$ de nudo a nudo y un trasmallo de $30 \times 3 \mathrm{~m}$ con una luz de malla de $5 \mathrm{~cm}$ de nudo a nudo, así como cuerdas y anzuelos. En cada estación se realizaron cuatro arrastres, dos en la parte central del río con la red agallera y dos a la orilla con el trasmallo. La red agallera se operó a una velocidad entre 1 y 2 nudos y un tiempo total de arrastre efectivo entre 20 y 30 minutos, por lo que las capturas son comparables en términos de abundancia relativa y no requieren de estandarización. La profundidad de captura varió entre $30 \mathrm{~cm}$ (orilla) y 3 metros (canal central). Todas las muestras de peces fueron identificadas en el campo mediante características anatómicas externas utilizando la clave propuesta por Bussing (1998). A cada ejemplar se le determinó la longitud total y estándar (precisión $0.5 \mathrm{~cm}$ ) y peso total (precisión $0.5 \mathrm{~g}$ ). Se calculó la frecuencia relativa (FR) de captura de acuerdo con la relación: FR = NVA/NTM, donde $\mathrm{NVA}=$ número de veces que aparece en los muestreos y NTM = número total de muestreos. Con base en este cálculo y una modificación de lo propuesto por Bravo-Núñez y Yáñez-Arancibia (1979), Yáñez-Arancibia et al. (1980) y Rojas et al (1994) se propone un arreglo comunitario de las comunidades de peces de acuerdo con su origen y frecuencia de aparición en los muestreos. En ese sentido se consideraron visitantes ocasionales o especies migratorias las especies con frecuencia $\leq 10 \%$, 
residentes estacionales o cíclicas aquellas especies con $11-30 \%$ y finalmente como residentes permanentes las especies con $31-100 \%$. Se estimaron índices ecológicos a nivel de especies y comunitarios (Shannon-Wiener (H'), Simpson (D) y Equitatividad (J)) (Henderson y Seaby 1998). La similitud entre las áreas muestreadas o heterogeneidad ambiental se calculó mediante el índice de Jaccard (Jij), definido por: Jij $=\mathrm{Cij} / \mathrm{Ci}+\mathrm{Cj}+\mathrm{Cij}$, donde $\mathrm{Cij}=\#$ de especies en ambas estaciones, $\mathrm{Ci}=$ Número de especies en la estación 1 y $\mathrm{Cj}$ = Número de especies en la estación 2. La relación entre las condiciones físicas (temperatura, oxígeno disuelto, lluvia y sólidos suspendidos y sólidos disueltos) versus el número de ejemplares y especies fueron determinadas mediante el coeficiente de correlación de Pearson (Conover 1971). Las especies se dividieron en primarias y secundarias de acuerdo con la definición propuesta por Miller (1966), Myers (1966) y Bussing (1988).

\section{RESULTADOS}

Condiciones climatológicas, características físico-químicas del río y caudales: $\mathrm{La}$ zona de estudio se encuentra bajo la influencia climática del Pacífico sur, caracterizada por variaciones en la distribución espacial y temporal de la precipitación. El régimen de precipitación tiene una marcada y bien definida época seca de diciembre a abril y una época lluviosa de mayo a noviembre. En la zona de estudio la precipitación promedio anual fue de $170.5 \mathrm{~m}^{3} / \mathrm{s}$, la precipitación más baja se registró en marzo $\left(27 \mathrm{~m}^{3} / \mathrm{s}\right)$. La temperatura del agua mostró una fuerte variación, de acuerdo con la estación climatológica de El Brujo, la temperatura del agua osciló entre $31.8^{\circ} \mathrm{C}$ (marzo) y $20.7^{\circ} \mathrm{C}$ (octubre) (Fig. 2). El pH se mantuvo ligeramente básico pero muy estable durante el año, con valores promedio de 7.6. Los valores de la demanda bioquímica de oxígeno (DBO) oscilaron entre 0.47 (agosto) y 1.62 (septiembre). El oxígeno disuelto presentó un valor promedio anual de $7.4 \mathrm{mg} / \mathrm{L}$. Durante abril 2004 se encontraron los valores más bajos de oxígeno disuelto (6.3) y el más alto en febrero (8.35). Para el 2004 el promedio anual fue de $328.7 \mathrm{~m}^{3} / \mathrm{s}$, mientras que para el 2005 fue de $343.2 \mathrm{~m}^{3} / \mathrm{s}$. En ambos períodos hay una clara reducción de caudales entre enero y abril (Fig. 3), con un incremento sostenido hasta su máximo en octubre (492 $\left.\mathrm{m}^{3} / \mathrm{s}-2004,531 \mathrm{~m}^{3} / \mathrm{s}-2005\right)$.

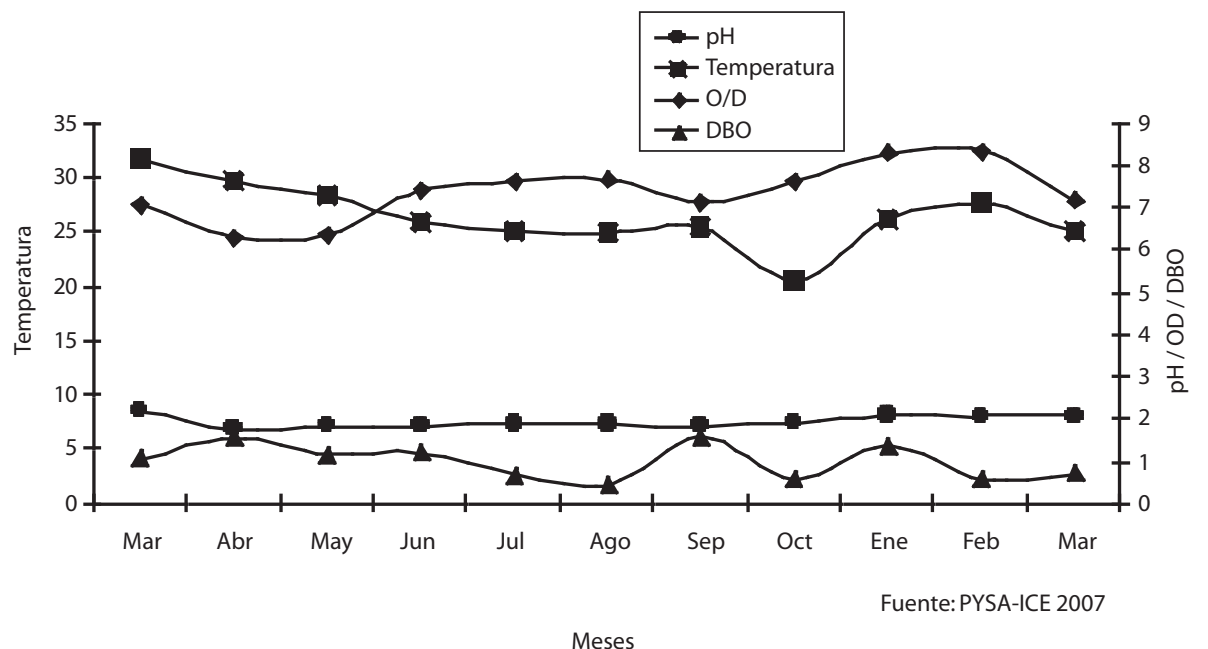

Fig. 2. Variación mensual de la temperatura, $\mathrm{pH}, \mathrm{OD}$ y $\mathrm{DBO}$ en la zona de estudio.

Fig. 2. Monthly variation of temperature, $\mathrm{pH}, \mathrm{DO}$ and $\mathrm{CDO}$ in the area of study. 


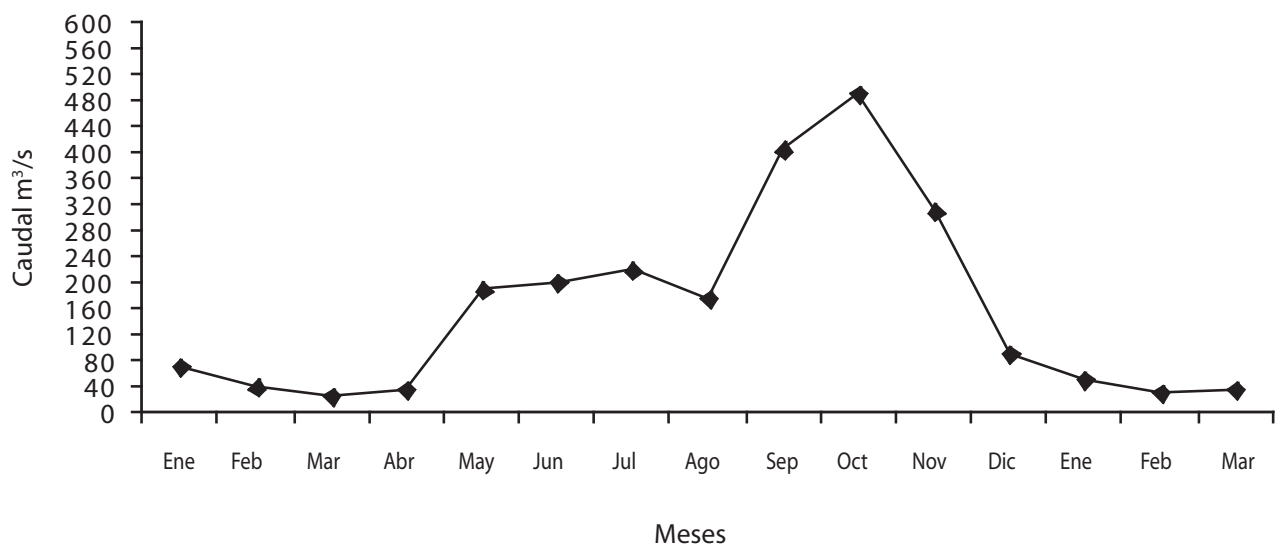

Fig. 3. Variación de los caudales del río Grande de Térraba, de enero 2004 a marzo 2005. Estación 1, El Brujo.

Fig. 3. Flow variation of river of Grande de Térraba, from January 2004 to March 2005. El Brujo sample station number one.

Estructura poblacional: Durante el período de estudio se capturaron 984 ejemplares, distribuidos en 14 familias, 26 géneros y 33 especies, de las cuáles tres fueron endémicas (Cuadro 1). Tróficamente la mayoría de las especies registradas correspondieron a carnívoras (48\%), un tercio omnívoras (33.3\%), 12\% detritívoros y sólo dos especies herbívoras exclusivas. La familia con mayor cantidad de especies fueron Cichlidae $(\mathrm{n}=5)$, Characidae $(\mathrm{n}=4)$ y Poecilidae $(\mathrm{n}=3)$. La machaca (Brycon behreae) fue la especie más abundante con 556 ejemplares, lo que corresponde a $56.5 \%$ de la captura (Cuadro 2). Con menores porcentajes aparecen las sardina Astyanax aeneus (7.2\%) y la sardinita Roeboides ilseae (6.3\%) (Cuadro 2). La riqueza ictiofaunística de la zona de estudio se complementó con especies que aportaron pocos ejemplares y en algunos casos sólo uno, por ejemplo el tepemechín Agonostomus monticola (Mugilidae), el lamearena Awaous transandeanus (Gobiidae), el jurel Caranx caninus (Carangidae), la guavina Dormitator latifrons (Eleotridae) y el pargo Lutjanus novemfasciatus (Lutjanidae). En ese Cuadro 2 se pueden visualizar el número de ejemplares, tallas y pesos de cada uno de las especies.

La biomasa total fue 147410.9 g., de la cuál el $53.7 \%$ la aportó la machaca $B$. behreae con 79127.3 g, seguido de el róbalo C. nigrescens con $30205.8 \mathrm{~g}$ (20.5\%), la tilapia $O$. niloticus (especie introducida) con $13984.3 \mathrm{~g}$ (9.5\%), otro róbalo C. nigrescens con 11118.0 (7.5\%) y el palmito E. brevimanus con 9377.9 (6.4\%). El mayor peso y talla correspondió a un ejemplar de róbalo $C$. nigrescens capturado en la estación 4, con un pesó de $1700 \mathrm{~g}$ y con 58 $\mathrm{cm}$. El ámbito más amplio de tallas y pesos correspondió a $B$. behreae con 3.4 y $46 \mathrm{~cm}$ y 0.70 a $1200 \mathrm{~g}$ respectivamente (Cuadro 2).

Sobre el origen de los peces hay dos familias estrictamente primarias Characidae y Loricariidae, hay un predominio de especies secundarias y de origen estuarino, entre ellos los jureles (C. caballus y C. caninus), los cuminates (C. steindachnerii y $C$. tuyra) y los pargos (L. jordani y L. novemfasciatus), estos últimos recolectados en la estación $1 \mathrm{y}$ 3 aproximadamente a $80 \mathrm{~km}$ río arriba de la boca del estuario. Con respecto a la riqueza de especies por estación, el mayor número de táxones $(\mathrm{N}=21,64 \%)$ fueron recolectadas en la estación 1 y la menor se presentó en la estación $2(\mathrm{~N}=7,21 \%)$. No se detectó un patrón en la distribución de tallas por sitios, ya que los individuos más grandes se capturaron en todas las estaciones de muestreo, igualmente la distribución general de ejemplares 
CUADRO 1

Familias, géneros y especies recolectadas, río Grande de Térraba, Costa Rica

TABLE 1

Families, genera and species collected in the river Grande de Térraba, Costa Rica

\begin{tabular}{|c|c|c|c|c|}
\hline Familia & Nombre científico & Nombre común & Grupo trófico & $\begin{array}{c}\text { Distribución } \\
\text { altitudinal } \\
\text { msnm }\end{array}$ \\
\hline ACHIRIDAE (2) & Trinectes fonsecensis & Hoja & Carnívoro (insectos acuáticos) & $0-55^{*}$ \\
\hline \multirow[t]{2}{*}{ ARIIDAE (2) } & Cathorops steindachnerii & Cuminate & Omnívoro & $0-55^{*}$ \\
\hline & C. tuyra & Cuminate & Omnívoro & $0-55^{*}$ \\
\hline \multirow[t]{2}{*}{ CARANGIDAE (2) } & Caranx caballus & Jurel & Carnívoro & $0-55^{*}$ \\
\hline & C. caninus & Bonito & Carnívoro & $0-55^{*}$ \\
\hline \multirow[t]{2}{*}{ CENTROPOMIDAE (2) } & Centropomus nigrescens & Robalo & Carnívoro & $0-145^{*}$ \\
\hline & C. viridis & Robalo & Carnívoro & $0-145^{*}$ \\
\hline \multirow[t]{4}{*}{ CHARACIDAE (1) } & Astyanax aeneus & Sardina & Omnívoro (semillas, frutos, insectos) & $10-145$ \\
\hline & Brycon behreae & Machaca & Omnívoro (semillas, frutos, insectos) & $10-640$ \\
\hline & Hyphessobrycon savegei & Sardina (endémica) & Carnívoro (insectos acuáticos) & $0-70$ \\
\hline & Roeboides ilseae & Sardina (endémica) & Carnívoro (escamas e insectos) & $10-660$ \\
\hline \multirow[t]{5}{*}{ CICHLIDAE (2) } & Archocentrus sajica & Mojarra (endémica) & Herbívoro (algas filamentosas) & $10-680$ \\
\hline & Astatheros diquis & Mojarra (endémica) & Omnívoro (insectos, frutas) & $16-700$ \\
\hline & A. altifrons & Mojarra & Carnívoro (insectos acuáticos) & $20-400$ \\
\hline & Oreochromis niloticus & Tilapia & Omnívoro (semillas, frutos, peces) & 145 \\
\hline & Theraps sieboldii & Mojarra & $\begin{array}{l}\text { Herbívoro (adultos), carnívoros } \\
\text { (juveniles) }\end{array}$ & $10-840$ \\
\hline \multirow[t]{2}{*}{ ELEOTRIDAE (2) } & Dormitator latifrons & Guavina & Omnívoro & $0-145^{*}$ \\
\hline & Gobiomorus maculatus & Guavina & Carnívoro (crustáceos y peces) & $0-145^{*}$ \\
\hline \multirow[t]{2}{*}{ GERREIDAE (2) } & Eugerres brevimanus & Palmito & Carnívoro (microbivalvos) & $0-145^{*}$ \\
\hline & Eucinostomus currani & Palmito & Carnívoro (larvas acuáticas) & $0-145^{*}$ \\
\hline \multirow[t]{2}{*}{ GOBIIDAE (2) } & Awaous transandeanus & Lamearena & Omnívoro & $0-20$ \\
\hline & Sycidium salvini & Chupa piedra & Omnívoro & $0-660$ \\
\hline HAEMULIDAE (2) & Haemulopsis leuciscus & Roncador & Carnívoro & $0-145^{*}$ \\
\hline LORICARIIDAE (1) & Hypostomus panamensis & Ruisaca & Detritívoro & $20-560$ \\
\hline \multirow[t]{2}{*}{ LUTJANIDAE (2) } & Lutjanus jordani & Pargo ñanguero & Carnívoro & $0-145^{*}$ \\
\hline & L. novemfasciatus & Pargo negro & Carnívoro & $0-95^{*}$ \\
\hline \multirow[t]{2}{*}{ MUGILIDAE (2) } & Agonostomus monticola & Tepemechín & Omnívoro & $0-650$ \\
\hline & Mugil curema & Lisa & Omnívoro & $0-145^{*}$ \\
\hline \multirow[t]{4}{*}{ POECILLIDAE (2) } & Poecilia gillii & Olomina & Detritívoro & $0-1220$ \\
\hline & P. mexicana & Olomina & Detritívoro & $0-145^{*}$ \\
\hline & Poeciliopsis elongata & Olomina & Detritívoro & $20-940$ \\
\hline & Priapichthys panamensis & Olomina & Carnívoro (insectívoro) & $0-95^{*}$ \\
\hline
\end{tabular}

El grupo trófico y la distribución latitudinal se presentan según Bussing (1998) y Fishbase (2006). * Nuevos registros altitudinales. (1) Especies de agua dulce primarias, (2) especies secundarias, de acuerdo con Miller (1966) y Myers (1966).

Trophic group and latitudinal distribution according to Bussing (1998) and Fishbase (2006). *New altitudinal register. (1) primary freshwater fishes, (2) secondary freshwater fishes, according with Miller (1966) and Myers (1966). 
CUADRO 2

Número de ejemplares, tallas y peso de la ictiofauna capturada, río Grande de Térraba, Costa Rica (orden alfabético)

TABLE 2

Number of samples, length and weight of ichthyofauna captured in the river Grande de Térraba, Costa Rica (alphabetic order)

\begin{tabular}{|c|c|c|c|c|c|c|c|c|}
\hline \multirow{2}{*}{ Especies } & \multirow{2}{*}{$\mathrm{N}$} & \multicolumn{2}{|c|}{ Long. Total $(\mathrm{cm})$} & \multicolumn{2}{|c|}{ Long. Est. (cm) } & \multicolumn{2}{|c|}{ Peso (g) } & \multirow[t]{2}{*}{$\begin{array}{l}\text { Biomasa } \\
\text { Total (g) }\end{array}$} \\
\hline & & Max & Min & Max & Min & Max & Min & \\
\hline Agonostomus monticola & 1 & \multicolumn{2}{|c|}{10.10} & \multicolumn{2}{|c|}{8.3} & \multicolumn{2}{|c|}{10.0} & 10.0 \\
\hline Archocentrus sajica & 19 & 10.6 & 2.5 & 20.8 & 0.2 & 8.4 & 2.0 & 55.2 \\
\hline Astatheros diquis & 5 & 4.8 & 3.8 & 3.2 & 3.0 & 3.8 & 2.8 & 15.4 \\
\hline Astatheros altifrons & 18 & 27.0 & 3.4 & 93.2 & 0.8 & 24.2 & 2.6 & 511.8 \\
\hline Astyanax aeneus & 71 & 10.2 & 2.1 & 12.1 & 0.10 & 8.2 & 1.4 & 281.6 \\
\hline Awaous transandeanus & 1 & \multicolumn{2}{|c|}{9.2} & \multicolumn{2}{|c|}{8.0} & 6 & & 6.0 \\
\hline Brycon behreae & 556 & 46.0 & 3.4 & 1200.0 & 0.7 & 42.0 & 3.0 & 79127.3 \\
\hline Caranx caballus & 3 & 13.2 & 11.3 & 26.0 & 20.0 & 11.3 & 9.4 & 71.0 \\
\hline Caranx caninus & 1 & \multicolumn{2}{|c|}{24.4} & \multicolumn{2}{|c|}{20} & 145 & & 147.0 \\
\hline Cathorops steindachnerii & 1 & \multicolumn{2}{|c|}{23} & \multicolumn{2}{|c|}{20} & 118 & & 118.0 \\
\hline Cathorops tuyra & 1 & \multicolumn{2}{|c|}{15.0} & \multicolumn{2}{|c|}{12.2} & 30.0 & & 30.0 \\
\hline Centropomus nigrescens & 64 & 58.0 & 17.0 & 1700.0 & 37.0 & 51.0 & 13.5 & 30205.8 \\
\hline Centropomus viridis & 14 & 49.5 & 16.2 & 1100.0 & 22.0 & 42.0 & 13.2 & 6021.0 \\
\hline Dormitator latifrons & 1 & \multicolumn{2}{|c|}{13.1} & \multicolumn{2}{|c|}{11.2} & 3.5 & & 123.0 \\
\hline Eucinostomus currani & 2 & 7.2 & 7.1 & 4.0 & 3.0 & 6.0 & 5.8 & 7.0 \\
\hline Eugerres brevimanus & 49 & 38.5 & 9.0 & 726.0 & 9.2 & 35.5 & 7.0 & 9377.9 \\
\hline Gobiomorus maculatus & 4 & 17.0 & 9.4 & 47.6 & 4.6 & 14.0 & 8.0 & 107.8 \\
\hline Haemulopsis leuciscus & 3 & 43.0 & 13.4 & 1020.0 & 15.0 & 38.0 & 12.0 & 1065.0 \\
\hline Hyphessobrycon savegei & 5 & 9.5 & 7.0 & 12.0 & 8.0 & 8.0 & 6.0 & 50.5 \\
\hline Hypostomus panamensis & 12 & 29.0 & 7.4 & 310.0 & 2.3 & 23.0 & 6.0 & 2579.0 \\
\hline Lutjanus jordani & 3 & 40.2 & 27.0 & 1128.6 & 286.0 & 34.0 & 22.0 & 1900.6 \\
\hline Lutjanus novemfasciatus & 1 & \multicolumn{2}{|c|}{27.0} & \multicolumn{2}{|c|}{22.0} & 230.0 & & 230.0 \\
\hline Mugil curema & 2 & 35.0 & 32.0 & 400.0 & 350.0 & 28.0 & 26.0 & 750.0 \\
\hline Oreochromis niloticus & 22 & 44.5 & 9.0 & 2074.0 & 7.5 & 37.0 & 7.0 & 13984.3 \\
\hline Poecilia gillii & 41 & 8.1 & 2.5 & 9.0 & 0.3 & 2.0 & 1.3 & 79.4 \\
\hline Poecilia mexicana & 2 & 5.0 & 4.0 & 2.0 & 0.5 & 4.0 & 3.0 & 18.0 \\
\hline Poeciliopsis elongata & 2 & 6.0 & 3.8 & 1.0 & 0.8 & 4.8 & 3.0 & 3.0 \\
\hline Priapichthys panamensis & 4 & 5.5 & 2.5 & 2.0 & 0.5 & 4.5 & 2.0 & 4.5 \\
\hline Roeboides ilseae & 62 & 12.8 & 3.0 & 24.5 & 0.2 & 10.6 & 2.3 & 277.8 \\
\hline Sycidium salvini & 10 & 13.2 & 5.8 & 24.1 & 2.2 & 10.8 & 4.8 & 102.0 \\
\hline Theraps sieboldii & 2 & 12.0 & 8.0 & 30.0 & 2.0 & 10 & 7 & 32.0 \\
\hline Trinectes fonsecensis & 2 & 17.0 & 8.0 & 110.0 & 9.0 & 6.60 & 15.0 & 119.0 \\
\hline
\end{tabular}


de tallas pequeñas en toda la zona de estudio, sin embargo cuando se analizó la distribución de las especies a lo largo del gradiente de estudio se notó la tendencia a dos patrones, el primero conformado por táxones ampliamente distribuidos en todas las estaciones, por ejemplo A. aeneus, B. behreae, $C$. nigrescens y $C$. viridis y el segundo por un conjunto de especies restringidas a una sola estación, entre ellas A. monticola (1), A. transandeanus (4) y C. caninus (3) (Cuadro 3). Respecto al número de especies la estación 1 fue la más diversa con un $64 \%$ de representación de los táxones de la zona de estudio, seguida por la estación 4 con 20 especies $(61 \%)$. La estación 2 presentó la menor cantidad de especies ( $\mathrm{N}=7$ especies) (Cuadro 3).

B. behreae fue la única especie capturada en todos los muestreos, sin embargo otros grupos de peces presentaron frecuencias de mas del 50\% anual de captura, entre ellas A. aeneus, $C$. nigrescens, E. brevimanus y $R$. ilseae. Otras solo fueron capturadas una vez, por ejemplo: $A$. diquis, $A$. transandeanus, $C$. steindachnerii, $D$. latifrons, $H$. savegei, P. mexicana y P. elongata (Cuadro 4). Temporalmente, junio registró la mayor cantidad de especies por muestreo (16), seguido por enero $(n=12)$ y febrero $(n=11)$.
Octubre fue el período con menor cantidad de especies observadas $(n=2)$. De acuerdo al análisis del componente comunitario, el $42 \%$ del conjunto ictiológico estuvo dominado por visitantes ocasionales o especies migratorias, mientras que las especies residentes cíclicas y residentes temporales presentaron un porcentaje similar (30 y 28\% respectivamente).

Los muestreos de mayo y julio aportaron la mayor cantidad de individuos y especies, mientras que octubre presentó la menor (Fig. 4). Se detectó un patrón general de mayor captura durante la época seca.

El número de especies y cantidad de ejemplares registrados en este estudio, reveló una disminución en la estación 1 (El Brujo), que coincidió con la transición climática. En términos generales, los meses con menor precipitación (enero-abril) aportan mayor cantidad de especies y de ejemplares (Fig. 5A). Por el contrario en la estación 2 (Coto) es en la época lluviosa donde se capturó mayor cantidad de especies e individuos. Incluso hay meses (enero, febrero, marzo y abril) en que en esta estación no se capturó nada (Fig. 5B). En la estación 3 (Lagarto) presentó baja diversidad y una tendencia a la reducción de especies durante la época lluviosa (Fig. 5C). En la estación 4

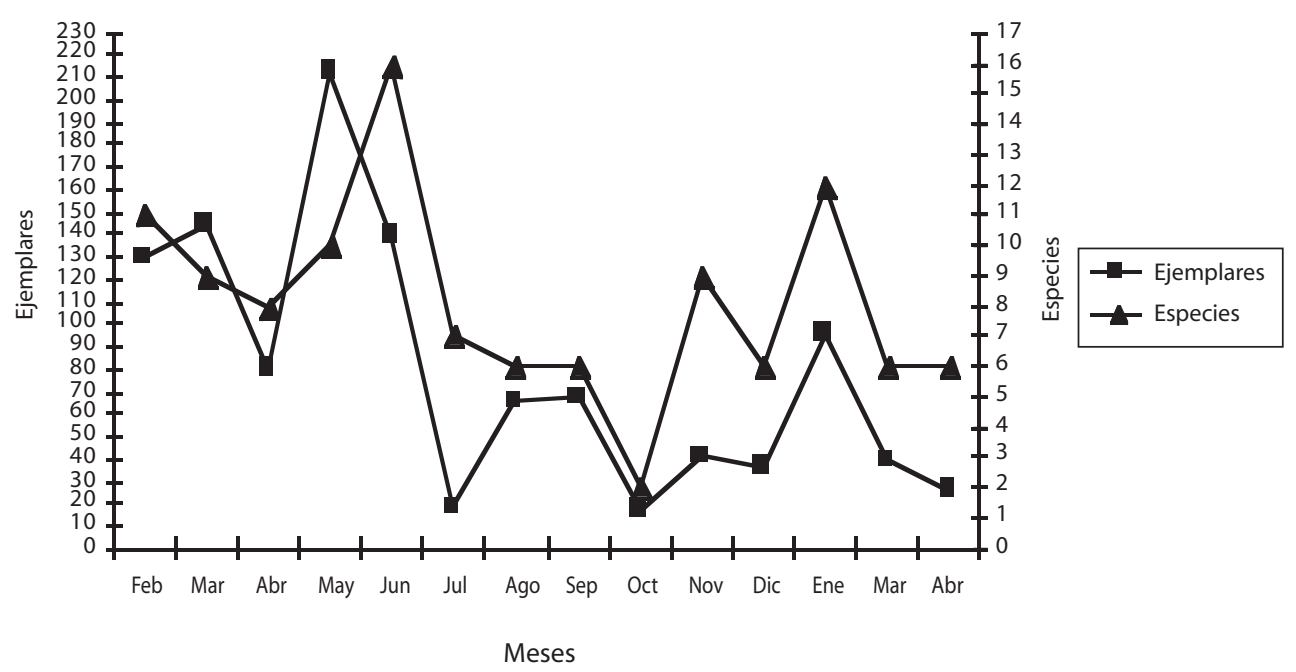

Fig. 4. Variación del número de especies y ejemplares capturados mensualmente, río Grande de Térraba, Costa Rica.

Fig. 4. Monthly variation in number of species and specimens, Térraba river, Costa Rica. 
CUADRO 3

Frecuencia de presencia por estación de la ictiofauna capturada en el río Grande de Térraba, Costa Rica

TABLE 3

Frequency of occurrence of fish captured in different sample stations of river Grande de Térraba, Costa Rica

Taxon

Agonostomus monticola

Archocentrus sajica

Astatheros diquis

Astatheros altifrons

Astyanax aeneus

Awaous transandeanus

Brycon behreae

Caranx caballus

Caranx caninus

Cathorops steindachnerii

Cathorops tuyra

Centropomus nigrescens

Centropomus viridis

Dormitator latifrons

Eucinostomus currani

Eugerres brevimanus

Gobiomorus maculatus

Haemulopsis leuciscus

Hyphessobrycon savegei

Hypostomus panamensis

Lutjanus jordani

Lutjanus novemfasciatus

Mugil curema

Oreochromis niloticus

Poecilia gillii

Poecilia mexicana

Poeciliopsis elongata

Priapichthys panamensis

Roeboides ilseae

Sycidium salvini

Theraps sieboldii

Trinectes fonsecensis

Total ( $\%$ de presencia)
Estación 1 Estación 2 Estación 3 Estación $4 \quad$ Total

X 1

X 2

$\mathrm{X}$

$\mathrm{X}$

$\mathrm{X}$

X

$\mathrm{X}$

$\mathrm{X}$

X

X

$\mathrm{X}$

$\mathrm{X}$

$\mathrm{X}$

$\mathrm{X}$

$\mathrm{X}$

$\mathrm{X}$

$\mathrm{X}$

$\mathrm{X}$

$\mathrm{x}$

X

$\mathrm{X}$

$\mathrm{X}$

$\mathrm{X}$

X

$\begin{array}{ll}\mathrm{X} & \\ \mathrm{X} & \\ \mathrm{X} & \\ \mathrm{X} & \\ & \mathrm{X} \\ \mathrm{X} & \mathrm{X} \\ \mathrm{X} & \\ \mathrm{X} & \end{array}$

X

$21(64 \%) \quad 7(21 \%)$
Frecuencia de presencia (\%)
25

50

25

50

100

25

100

25

25

25

25

100

100

25

25

75

50

25

25

25

50

25

25

75

50

25

25

50

75

50

25

50 
CUADRO 4

Frecuencia de presencia anual de la ictiofauna capturada en el río Grande de Térraba, Costa Rica

TABLE 4

Annual frequency of occurrence of ichthyofauna captured in river Grande de Térraba, Costa Rica

\begin{tabular}{|c|c|c|c|c|c|c|c|c|c|c|c|c|c|c|c|c|c|}
\hline \multirow[t]{2}{*}{ Especies } & \multicolumn{14}{|c|}{ Meses } & \multirow[t]{2}{*}{ Total } & \multirow{2}{*}{$\begin{array}{c}\text { Presencia } \\
(\%)\end{array}$} & \multirow{2}{*}{$\begin{array}{l}\text { Component } \\
\text { comunitaric }\end{array}$} \\
\hline & $\mathrm{F}$ & M & $\mathrm{A}$ & M & $\mathrm{J}$ & $\mathrm{J}$ & A & $\mathrm{S}$ & $\mathrm{O}$ & $\mathrm{N}$ & $\mathrm{D}$ & $\mathrm{E}$ & M & A & & & \\
\hline Agonostomus monticola & & & & $\mathrm{X}$ & & & & & & & & & & & 1 & 7 & VO-M \\
\hline Archocentrus sajica & $\mathrm{X}$ & $\mathrm{X}$ & & & $\mathrm{X}$ & & & & & & & $\mathrm{X}$ & & & 4 & 28 & RE-C \\
\hline Astatheros diquis & & & $\mathrm{X}$ & & & & & & & & & & & & 1 & 7 & VO-M \\
\hline Astatheros altifrons & $\mathrm{X}$ & $\mathrm{X}$ & & & $\mathrm{X}$ & & & & & & & $\mathrm{X}$ & $\mathrm{X}$ & & 5 & 36 & $\mathrm{RP}$ \\
\hline Astyanax aeneus & & & & $\mathrm{X}$ & $\mathrm{X}$ & & $X$ & $\mathrm{X}$ & & & $\mathrm{X}$ & $X$ & $X$ & & 7 & 50 & $\mathrm{RP}$ \\
\hline Awaous transandeanus & & & & $\mathrm{X}$ & & & & & & & & & & & 1 & 7 & VO-M \\
\hline Brycon behreae & $\mathrm{X}$ & $\mathrm{X}$ & $\mathrm{X}$ & $\mathrm{X}$ & $\mathrm{X}$ & $\mathrm{X}$ & $X$ & X & $\mathrm{X}$ & $\mathrm{X}$ & $\mathrm{X}$ & $\mathrm{X}$ & $\mathrm{X}$ & $\mathrm{X}$ & 14 & 100 & $\mathrm{RP}$ \\
\hline Caranx caballus & & $\mathrm{X}$ & & & & & & & & & & & & & 1 & 7 & VO-M \\
\hline Caranx caninus & $X$ & & & & & & & & & & & & & & 1 & 7 & VO-M \\
\hline Cathorops steindachnerii & & & & & & & $X$ & & & & & & & & 1 & 7 & VO-M \\
\hline Cathorops tuyra & & & & & & & $\mathrm{X}$ & & & & & & & & 1 & 7 & VO-M \\
\hline Centropomus nigrescens & & & $\mathrm{X}$ & & $\mathrm{X}$ & & & & & $\mathrm{X}$ & & & & & 4 & 28 & $\mathrm{RE}-\mathrm{C}$ \\
\hline Centropomus viridis & & $X$ & & & $\mathrm{X}$ & $\mathrm{X}$ & & & & $\mathrm{X}$ & & & & & 4 & 28 & RE-C \\
\hline Dormitator latifrons & $X$ & & & & & & & & & & & & & & 1 & 7 & VO-M \\
\hline Eucinostomus currani & & & & $\mathrm{X}$ & & & & & & & & & & & 1 & 7 & VO-M \\
\hline Eugerres brevimanus & & & $\mathrm{X}$ & & $\mathrm{X}$ & & $\mathrm{X}$ & $\mathrm{X}$ & $\mathrm{X}$ & $\mathrm{X}$ & $\mathrm{X}$ & $\mathrm{X}$ & & $\mathrm{X}$ & 9 & 64 & $\mathrm{RP}$ \\
\hline Gobiomorus maculatus & $\mathrm{X}$ & & & & $X$ & X & & & & & & $\mathrm{X}$ & & & 4 & 28 & RE-C \\
\hline Haemulopsis leuciscus & & $\mathrm{X}$ & & & & & & & & & & & & & 2 & 14 & RE-C \\
\hline Hyphessobrycon savegei & & & & & $\mathrm{X}$ & & & & & & & & & & 1 & 7 & VO-M \\
\hline Hypostomus panamensis & & & $\mathrm{X}$ & & $\mathrm{X}$ & & & $\mathrm{X}$ & & $\mathrm{X}$ & & & & & 4 & 28 & RE-C \\
\hline Lutjanus jordani & & & & & & $\mathrm{X}$ & & & & $\mathrm{X}$ & $X$ & $\mathrm{X}$ & & & 4 & 28 & RE-C \\
\hline Lutjanus novemfasciatus & & & $\mathrm{X}$ & & & & & & & & & & & & 1 & 7 & VO-M \\
\hline Mugil curema & & & & & $\mathrm{X}$ & & & & & & & & & & 1 & 7 & VO-M \\
\hline Oreocromis niloticus & $X$ & $\mathrm{X}$ & & & & & & & & $\mathrm{X}$ & $\mathrm{X}$ & $\mathrm{X}$ & & $\mathrm{X}$ & 6 & 43 & $\mathrm{RP}$ \\
\hline Poecilia gillii & $\mathrm{X}$ & $X$ & & $X$ & & $X$ & & & & & & $X$ & $X$ & & 6 & 43 & $\mathrm{RP}$ \\
\hline Poecilia mexicana & $\mathrm{X}$ & & & & & & & & & & & & & & 1 & 7 & VO-M \\
\hline Poeciliopsis elongata & & & & $\mathrm{X}$ & & & & & & & & & & & 1 & 7 & VO-M \\
\hline Priapichthys panamensis & & & & $\mathrm{X}$ & $X$ & & & & & & & & & & 2 & 14 & RE-C \\
\hline Roeboides ilseae & & $X$ & $X$ & & $\mathrm{X}$ & & $X$ & $\mathrm{X}$ & & $X$ & & $X$ & $X$ & & 8 & 57 & $\mathrm{RP}$ \\
\hline Sycidium salvini & $\mathrm{X}$ & & & $\mathrm{X}$ & $\mathrm{X}$ & & & & & & & $\mathrm{X}$ & $\mathrm{X}$ & & 5 & 36 & $\mathrm{RP}$ \\
\hline Theraps sieboldii & $X$ & & & & $\mathrm{X}$ & & & & & & & & & & 2 & 14 & RE-C \\
\hline Trinectes fonsecensis & & & $\mathrm{X}$ & & & $\mathrm{X}$ & & & & & & & & $X$ & 3 & 21 & $\mathrm{RE}-\mathrm{C}$ \\
\hline
\end{tabular}

Total

$\begin{array}{llllllllllllll}11 & 9 & 8 & 10 & 16 & 7 & 6 & 6 & 2 & 9 & 6 & 12 & 6 & 6\end{array}$

$0-10 \%=$ VO-M, Visitantes ocasionales, migratorias o especies raras, $11-30 \%=$ RE-C, Residentes estacionales o cíclicas y $31-100 \%=\mathrm{RP}$, Residentes permanentes).

$(0-10 \%=$ VO-M, Occasional visitor, migratory or rare species, $11-30 \%=\mathrm{RE}-\mathrm{C}$, Resident species, stationary or cyclic and $31-100 \%=$ RP, Permanent resident species). 

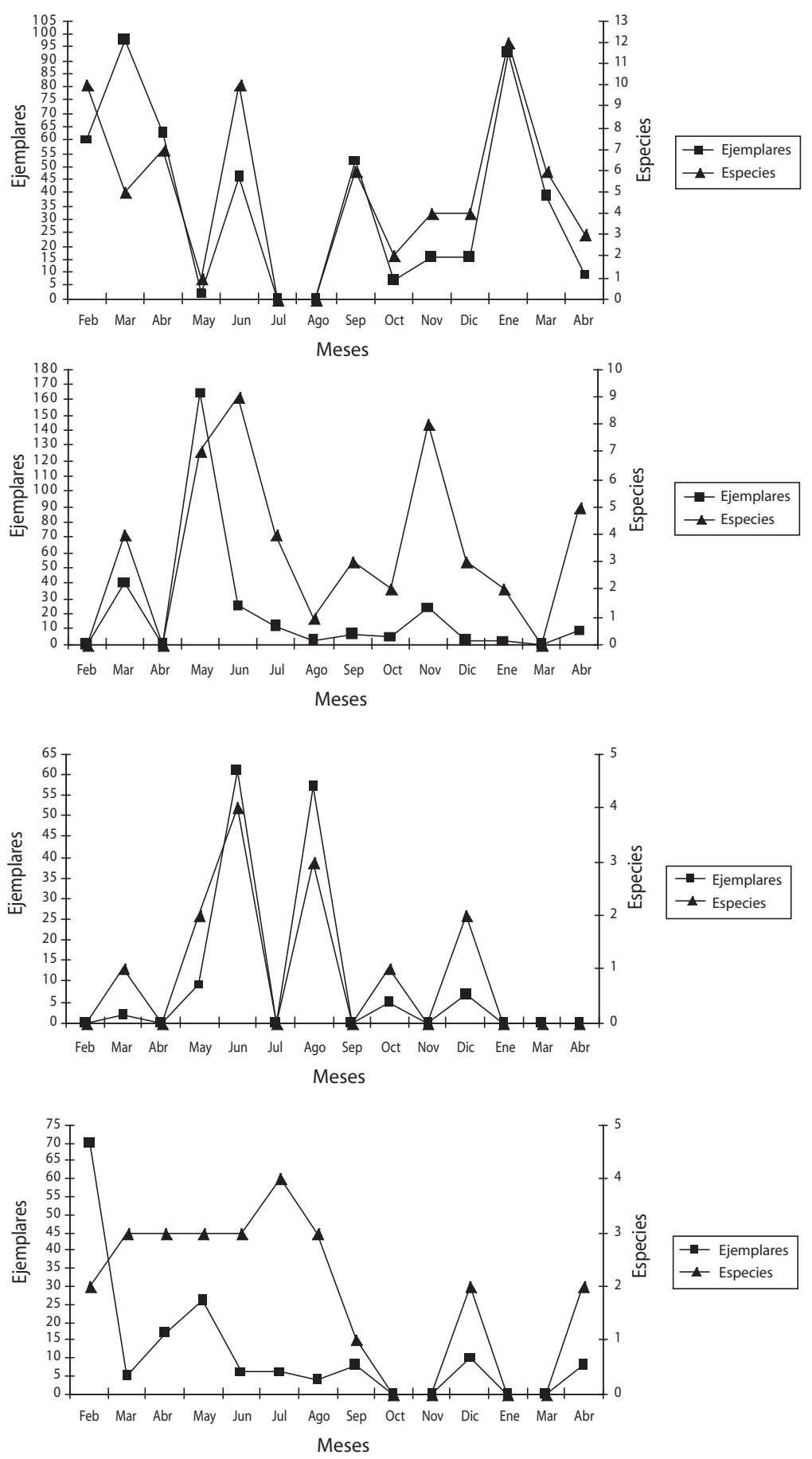

Fig. 5. Variación del número de especies y ejemplares capturados mensualmente en la estación El Brujo (A.), Coto (B.), Lagarto (C.) y Palmar (D.).

Fig. 5. Monthly variation of number of species and specimens captured, El Brujo (A.), Coto (B.), Lagarto (C.) y Palmar (D.) stations. 
se notó un aumento en la riqueza de especies y ejemplares en el verano (Fig. 5D).

Índices ecológicos: La diversidad ( $\left.\mathrm{H}^{\prime}\right)$ total en la zona de estudio fue de 1.84. Espacialmente la mayor diversidad se registró en la estación El Brujo (H’2.32), mientras que en Coto se presentó la menor (H' 1.67) (Fig. 6A). En Lagarto se presentó el valor más alto de diversidad comunitaria $(\mathrm{D}=13.54)$ y en Coto la menor ( $\mathrm{D}=7.1)$ (Fig. 6B).

Con respecto al índice de Equidad o Igualdad $(\mathrm{J})$, se observa que la estación El
Brujo presentó el valor más alto en la equitatividad de especies $(\mathrm{J}=0.88)$, mientras que el menor valor lo presentó la estación dos $(\mathrm{J}=0.63)$ (Fig.6C).

De acuerdo con el índice Jaccard (Cuadro 5), las estaciones más similares entre si fueron las estaciones uno con cuatro $(\mathrm{J}=0.43)$ y dos con tres $(\mathrm{J}=0.40)$. No se detectó correlación entre el número de especies y las variables físico-química, sin embargo, la cantidad de individuos se correlacionó positivamente con la temperatura $\left(\mathrm{R}^{2} 0.58\right)$ y el oxígeno disuelto ( $\mathrm{R}^{2}$ 0.61) (Cuadro 6).

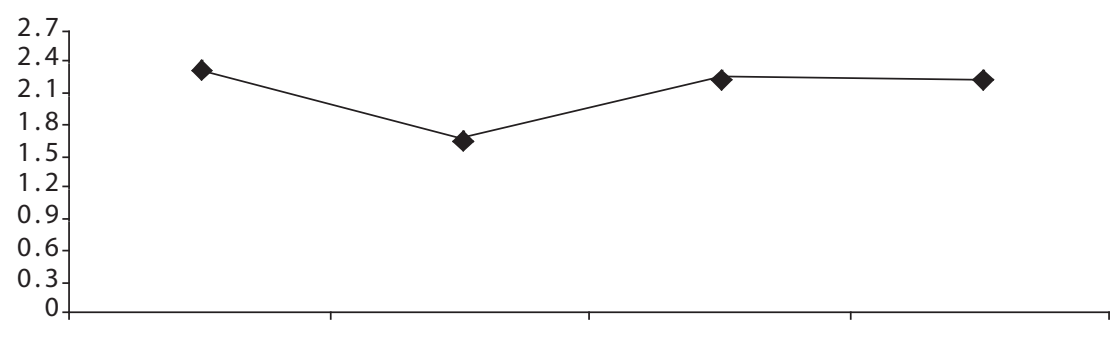

$\begin{array}{llll}\text { EST. } 1 & \text { EST. } 2 & \text { EST. } 3 & \text { EST. } 4\end{array}$

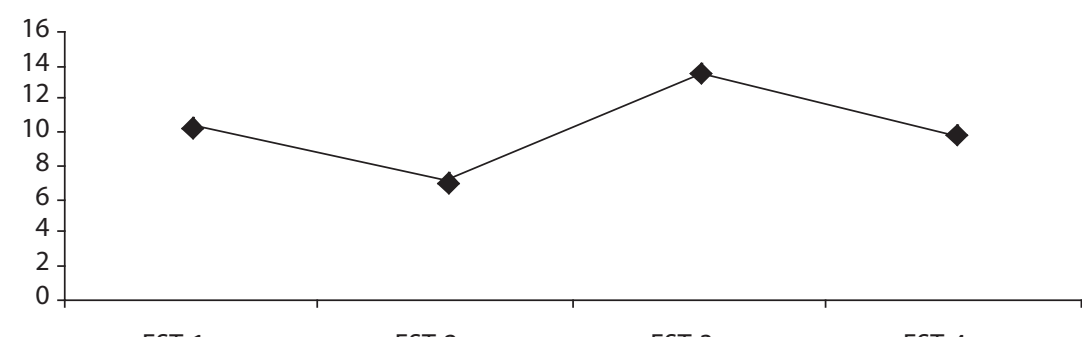

$\begin{array}{llll}\text { EST. } 1 & \text { EST. } 2 & \text { EST. } 3 & \text { EST. } 4\end{array}$

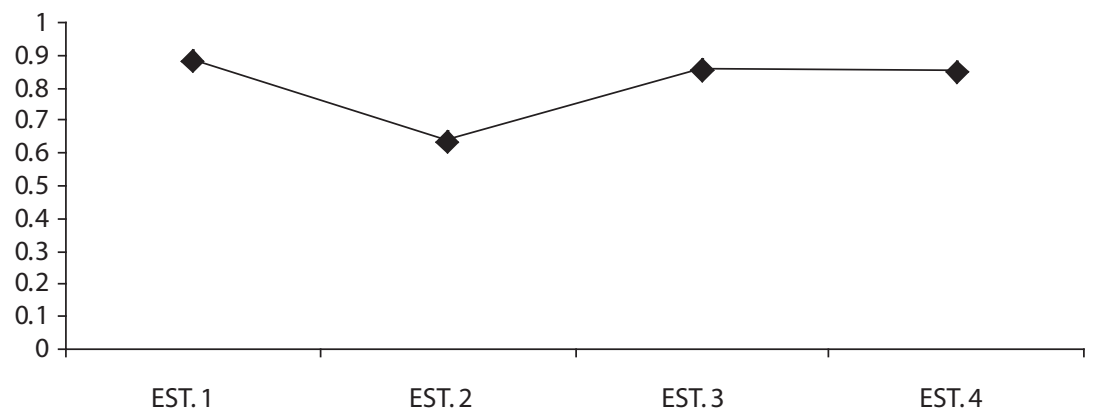

Fig. 6. Variación de los índices de diversidad de Shannon-Wiener (A), Simpson (B) y Equitatividad (C) en las estaciones de estudio.

Fig. 6. Variation of Shannon-Wiener (A), Simpson (B) and Evenness (C) indexes in the sample stations. 
CUADRO 5

Índice de similitud (Jaccard) en las estaciones de estudio.

TABLE 5

Evenness index (Jaccard) in the sample stations

$\begin{array}{ccccc}\text { Estaciones } & 1 & 2 & 3 & 4 \\ 1 & 1 & 0.27 & 0.30 & 0.43 \\ 2 & 0.27 & 1 & 0.40 & 0.29 \\ 3 & 0.30 & 0.40 & 1 & 0.31 \\ 4 & 0.43 & 0.29 & 0.31 & 1\end{array}$

\section{DISCUSION}

Con base en los últimos inventarios ictiofaunísticos, en Costa Rica, se han descrito aproximadamente 135 especies de peces de agua dulce (Bussing 1998), un 25\% ( $\mathrm{n}=33)$ fueron reconocidas en la zona de estudio. La mayor parte de este conjunto de especies, cumplen su ciclo de vida en agua dulce (potamódromas), sin embargo hay algunas especies periféricas de tipo catádromo, como el tepemechín Agonostomus monticola, que requieren, de acuerdo con Cruz (1987) y Bussing (1998), migrar al estuario a reproducirse. Otros grupos detectados fueron los visitantes anfídromos que sin mediar reproducción u otra actividad ontogénica crítica se desplazan entre el estuario y las aguas dulces. Se detectó dos ejemplos con desplazamiento anfídromo extremo (ambos róbalos $C$. nigrescens, $C$. viridis), recolectadas a $80 \mathrm{~km}$ del estuario. Los hallazgos sobre el ingreso de especies estuarinas a aguas dulces evidencian un cambio en la composición ictiofaunística reportada por Bussing (1998) en la riqueza taxonómica del tramo estudiado, ya que los registros de Caranx caballus, C. caninus, Cathorops steindachneri, Centropomus nigrescens, $C$. viridis, Haemulopsis leuciscus, Lutjanus jordani, L. novemfasciatus y Mugil curema, en el presente estudio, aumenta la cantidad de especies. La presencia de pece estuarinos, a distancias considerables del mar, confirman lo indicado por Lyons y Schneider (1990), que la distancia es una variable que podría explicar, no sólo los patrones longitudinales y la distribución de peces en el área de estudio, sino la estructura trófica del tramo estudiado.

La única especie introducida, reconocida en este estudio, fue la tilapia Oreochromis niloticus y aunque no se conoce con exactitud la fecha de inroducción, es evidente que ha sido exitosa en la invasión de esta cuenca, incluyendo la zona costera a lo largo del manglar de Térraba, donde ha sido atrapada frecuentemente (Rojas y Rodríguez obs. per.). Este avance de la tilapia desde el río hasta la costa repite el patrón observado por Pizarro y Rojas (1993) en el sector costero de Bebedero, Golfo de Nicoya, Pacífico de Costa Rica y por Oro y

CUADRO 6

Correlación entre parámetros físico-químicos y diversidad para la zona de estudio.

Fuente: Proyectos y Servicios Asociados (ICE)

TABLE 6

Correlation between fish species diversity and physical-chemical habitat characteristics. Source: Proyectos y Servicios Asociados (ICE)

Parámetro

$\mathrm{pH}$

Temperatura $\mathrm{C}^{\mathrm{o}}$

Oxígeno disuelto (mg/L)

Sólidos suspendidos (mg/L)

Sólidos disueltos $(\mathrm{mg} / \mathrm{L})$

Lluvia $\left(\mathrm{m}^{3} / \mathrm{s}\right)$
Número de especies

0.225

0.246

0.225

$-0.03$

$-0.358$

$-0.301$
Número de ejemplares

0.497

0.580

0.610

0.180

0.190

$-0.410$ 
Cabrera (1993) en el Caribe. La presencia de esta especie en la zona de manglar, es preocupante por la plasticidad alimentaria, migratoria y reproductiva, así como la capacidad de este cíclido para desplazar especies autóctonas. Por lo que es previsible un impacto negativo, no sólo en la ictiofauna, sino del equilibrio que existe con la biota asociada al río-manglar.

La ausencia de especies endémicas, tales como Bryconamericus terrabensis, Astatheros diquis, Brachyrhaphis rhabdophora, B. terrabensis, Piabucina boruca, Poeciliopsis retropinna, Pseudocheirodon terrabae, Pterobrycon myrnae y Rivulus uroflammeus, reportadas en la zona de estudio por Bussing (1998), puede ser explicada por la especificidad geográfica en que se encuentran (Winemiller 1983, Lyons y Schneider 1990) o por que el arte de pesca utilizado no permitió su captura debido al tamaño relativamente pequeno que alcanzan (aproximadamente $10 \mathrm{~cm}$ en Longitud total) (Bussing 1998, Henderson y Seaby 1998); por lo que para futuras investigaciones se recomienda dirigir los muestreos en los sitios designados como la localidad tipo. Un escenario mas pesimista podría ser que las especies endémicas pueden estar siendo afectadas por la degradación que esta afectando este río, producto del aumento de contaminantes (plaguicidas, plaguicidas, herbicidas), sedimento particulado y en suspensión, derivado de practicas agrícolas extensivas e industriales.

Sobre la identidad ictiofaunística de la zona de estudio existe afinidad con la Provincia Ictiológica Ístmica (Bussing 1976, 1985b y 1998 sensu stricto), en este sentido, y en concordancia con lo indicado por Myers (1966) y Bussing (1985b), la ictiofauna actual del río Grande de Térraba resulta de la combinación de procesos de dispersión, facultados por el intercambio biótico entre Norte y Sur América. Ictiogeográficamente este río tiene una posición particular, ya que constituye un hito geográfico que funciona como una zona de enlace y puente migratorio de peces dulceacuícolas de Norte y sur América, lo que sumado a sus características orográficas, geológicas, geomorfológicas y climáticas, la zona de estudio reúne una serie de condiciones que justifican ser una de las zonas de mayor endemismo de Costa Rica, así como el reconocimiento de ser un área de transición que faculta la presencia de antiguos y nuevos elementos ícticos americanos.

Los antiguos elementos representados por miembros de las familias Poecilidae, Characidae y Cichlidae, fueron frecuentes en nuestras capturas, condición que coincide con Myers (1966), Bussing (1976, 1985b) y Briggs (1984), en cuanto al carácter de amplia dispersión que se ve reflejado en la distribución en toda Centroamérica y parte de México. En contraste los nuevos elementos están restringidos a la Provincia Ístmica y por lo general no se extienden más allá de Punta Mala (Costa Rica), que según Bussing (1985b) coincide con el límite entre las Provincias Chiapas-Nicaragua e Ístmica. Los llamados nuevos elementos tienen una estrecha relación evolutiva con peces de la zona Pacífica de Panamá e incluso Colombia, de ahí que cuando se examinan las relaciones ictiológicas de los peces de la cuenca del Térraba hay que vincularlos mas con estos países que con zonas del Pacífico Central de Costa Rica. La conquista de elementos sureños estuvo caracterizada, según Bussing (1976, 1985b, 1998), por cuatro grupos de especies, de los dos primeros no hay táxones en el Térraba, del tercer grupo sólo incluye el género Aequidens, reportado para el río Coto (Bussing 1998) y finalmente del cuarto grupo, presentes en el Térraba, se reconocen los géneros Pterobrycon e Hypostomus.

El patrón de distribución de la ictiofauna en el tramo estudiado concuerda con las deducciones de Allan (1995) y Roldán (1992), de que en los ríos, desde su nacimiento hasta la desembocadura, se generan una serie de cambios geomorfológicos y modificaciones fisicoquímicos del agua, que no sólo permiten el establecimiento de comunidades ictiológicas adaptadas a hábitats particulares, sino de la dinámica de adición, sustracción y sustitución de especies. También es de esperarse que lo expresado por Vannote et al. (1980), Gilliam et al. (1993) y Jackson y Harvey (1989), sobre la estructura de las comunidades de peces a 
lo largo de ríos tropicales y templados, ocurra en el Térraba; particularmente sobre la alternancia de grupos específicos a medida que se desciende en altitud y una mayor complejidad estructural y funcional, expresada en términos de una mayor riqueza y diversidad de especies, en el sector de transición entre las comunidades de aguas dulces y marinas.

Para el caso del tramo estudiado era de esperarse que los $55 \mathrm{~km}$ que separan la estación uno de la cuatro y los $86 \mathrm{~km}$ que hay entre la primera estación y el estuario, así como los atributos geomorfológicos del tramo estudiado (ancho, profundidad y volumen de las pozas, cantidad de la cobertura presente y diversidad de sustrato) podrían resultar distancias suficientes como para generar condiciones ecológicas específicas y justificar diferentes agrupamientos ictiofaunísticos, así como una alternancia de especies en sentido este-oeste en dirección de flujo del río y hacia el manglar de Térraba. Sin embargo, no se descarta lo señalado por Lyons y Schneider (1990), de que la distancia al estuario es otra variable que podría ayudar a explicar el porque los táxones de origen estuarino parecen agruparse en los sectores tres y cuatro más próximas al manglar. Contrario a lo observado por Bussing y López (1977), en el tramo estudiado con excepción del caudal, no aparece un predictor (tamaño del río, interacción de especies) que permita asociar un grupo específico de especies con un punto de muestreo particular, de hecho los bajos índices de similitud resultan una señal de la poca afinidad entre zonas.

Para el tramo estudiado la sustitución de especies probablemente ocurra más por discontinuidades abruptas en la geomorfología y aumento de complejidad estructural (expresada en hábitat susceptibles de colonizar como pozas, remansos y orillas con vegetación), que por condiciones abióticas, evidenciado por la baja correlación que se obtuvo entre los parámetros físico-químicos y la diversidad de especies y ejemplares (Cuadro 6). Con excepción de una correlación media que se obtuvo entre el número de ejemplares y el oxígeno disuelto ( 0.61$)$, no se logró detectar una estrecha relación entre la riqueza de especies y las variables temperatura, $\mathrm{pH}$, sólidos suspendidos, disueltos o la lluvia. Probablemente el sector analizado se comporte de la forma en que lo definió Schiemer (2000) en donde las características ecológicas (depredación, sustitución de especies, niveles tróficos), no son las únicas predictoras de la estructura ictiogeográfica, sino que existe un intercepto entre la ecología, geomorfología e hidrología, de tal forma que las fluctuaciones en los niveles del agua conducen a un continuo cambio de la posición de los microhábitats y determina la disponibilidad, conexión y calidad de los refugios susceptibles de ser ocupados por los peces. Ese escenario es quizás el que más comúnmente ocurra en el Térraba debido a los cambios en el caudal que se dan entre la época seca y lluviosa.

El desvío del patrón normal, en la distribución y abundancia de peces, podría estar asociada con el estado de deterioro de la cuenca. Los bosques aledaños al tramo estudiado y encargados de aportar semillas, hojas $\mathrm{y}$ frutos, han sido transformados en cultivos de piña (Ananas sativus). Consecuentemente hay una evidente reducción de aportes alóctonos a la dieta de especies herbívoras, lo que podría explicar la poca presencia (sólo una especie, A. sajica) que, según Bussing (1998), consume materia vegetal. Otra posible explicación es que los desarrollos agrícolas impacten negativamente las comunidades ícticas, fundamentalmente por el aporte excesivo de contaminantes (fungicidas, plaguicidas y herbicidas) y por la pérdida de cobertura vegetal. De hecho Bojsen y Barriga (2002), aunque reconocieron que hay poca información sobre el impacto de la deforestación en la diversidad y estructura comunitaria de peces de ríos tropicales, son enfáticos en que existe un efecto inverso entre riqueza de peces, deforestación y/o aporte directo de nutrientes en forma de hojas, flores y frutos. Los resultados del presente estudio, parecen ajustarse a este modelo, porque evidentemente hay heterogeneidad en la composición de especies que resulta de la alteración de la cobertura vegetal en el tramo estudiado y segundo por la ausencia de especies raras, 
endémicas y con una alta inclinación a vivir en sectores restringidos, debidas a la deforestación de la zona de estudio. En contraste, la ictiofauna del Térraba no parece cumplir con un segundo supuesto de Bojsen y Barriga (2002), de que en ríos con condiciones reforestadas abunden especies omnívoras y/o insectívoras y que en zonas deforestadas existan especies que consuman perifiton. La zona de estudio, aunque es deforestada, alberga especies cuyos hábitos alimentarios son fundamentalmente de hojas, frutos, insectos o ambas. Esta desviación del patrón de Bojsen y Barriga (2002) quizás pueda explicarse por la lluvia, deriva y arrastre de nutrientes que se ocasiona desde las partes altas de la cuenca, sin embargo esta hipótesis debe ser corroborada.

Sobre los patrones de riqueza y diversidad de especies, los resultados de esta investigación son intermedios con respecto a los reportados en zonas cercanas y ecosistemas similares de Costa Rica, por ejemplo Winemiller (1983) reportó para Corcovado 40 especies, para un $25 \%$ de especies estuarinas. Estimaciones semejantes para el sector de Sirena, fueron realizadas por Winemiller y Morales (1989). Cuando se compara los valores de estos índices con los obtenidos en otras zonas del país, se concluye que el río Grande Térraba presenta una diversidad intermedia con respecto a ríos del sector de Puntarenas y Guanacaste (Bussing 1998 y Alpírez 1985), pero mayor que la de ríos de la vertiente Norte, como el río Frío y si la comparación se hace en ríos que desembocan en el Caribe, se observó que el Térraba tiene mayor riqueza, por ejemplo el caso del río Caño Negro (Sáenz et al. 2006).

Las semejanzas y diferencias entre los patrones de diversidad y abundancia de peces en el río Grande de Térraba y otros ecosistemas tropicales locales o regionales, conllevan a una interrogante de fondo: ¿Cómo se regula localmente el número de especies?. Una hipótesis apoyada en los supuestos de Menge y Olson (1990) y Ricklefs y Schluter (1993) sugiere, que mas que condiciones puntuales, podrían existir una combinación azarosa de escalas multi espaciales (local contra regional) y temporales (contemporánea contra histórica), así como un arreglo de factores abióticos-bióticos que organizan, limitan y ordenan la ictiodiversidad, por lo que se debe tomar en cuenta las sugerencias de Ricklefs (1987) y Angermeier y Winston (1988) de considerar la importancia relativa de los procesos de mega escala (por ejemplo la dispersión), los cuáles generalmente determinan la posibilidad de presencia local, así como los eventos de pequeña escala (competencia, disturbio), lo que limita temporalmente el número de especies que ocurren.

Consideraciones finales: La presente investigación es pionera en intentar describir la dinámica ictiológica del río Grande de Térraba, uno de los ecosistemas lóticos más grandes, caudalosos y alterados de Costa Rica. El enfoque del estudio, basado en muestreos sistemáticos geográficamente distribuidos, complementa los hallazgos taxonómicos sintetizados por Bussing (1998) y pone a disposición una línea base actualizada para la toma de decisiones sobre el manejo y conservación de este río. Los resultados se apoyan en publicaciones recientes y en la comparación con ecosistemas similares, condición que permite establecer puntos de comparación y proponer análisis de posibles escenarios de impactos y afectaciones, sobre todo cuando consideramos la conexión natural que existe con el manglar.

Este aporte resulta esencial para cualquier propuesta de manejo ecológico y se convierte en un instrumento de gestión ambiental estratégica para iniciativas, como las de generación hidroeléctrica, donde es obligatorio el desvío de aguas, alteración de cauces y flujos y la construcción de represas. Esta investigación armoniza con la política ambiental institucional en específico con planificar y ejecutar obras con fundamento en el principio de desarrollo sostenible y con una gestión articulada por la conservación, protección, recuperación y uso responsable del ambiente.

Evidentemente conocer para manejar es la mejor estrategia para alentar el progreso ambientalmente sostenible y es una meta que el Instituto Costarricense de Electricidad 
promueve mediante el desarrollo de este tipo de investigaciones. Se espera que los hallazgos permitan establecer parámetros que orienten propuestas de gestión ambiental integral especialmente dirigidas a la restauración ecológica de ríos y cauces, manejo de desembalses, relaciones con la comunidad, evaluaciones ecológicas de pre y factibilidad, estudios de impacto ambiental y educación ambiental de temas relacionados con ríos, es una tarea que no debe postergarse.

\section{AGRADECIMIENTOS}

El desarrollo de esta investigación fue posible gracias al apoyo de muchas personas. En forma especial se reconoce la ayuda de Gilberto de la Cruz y Roberto Jiménez Gómez (CENPE-ICE), quienes apoyaron el desarrollo de este estudio. A Lorena Mariño y Frank Garro (CENPE-ICE) por su ayuda en el manejo de bases de datos y estadística. A Marco Tapia y Francisco Rodríguez por el apoyo y facilidades brindadas en el campamento de Boruca y Luis Salazar Ferrero por el apoyo en el campo. A José Francisco Fernández por la información sobre calidad de agua, a Sadí Laporte por los datos climatológicos, a Jorge Picado por sugerencias y María José Ocón y José Luis Aguirre por su apoyo en el SIG.

\section{RESUMEN}

Desde el inicio de la estación seca del 2004 hasta la estación lluviosa de 2005, se llevó a cabo una investigación, con el objetivo de determinar la diversidad, abundancia y patrones de distribución de las comunidades de peces de agua dulce del río Grande de Térraba. El muestreo de peces se llevó a cabo utilizando una combinación de artes de pesca, entre ellas las redes agalleras, redes finas y observaciones visuales. Fueron recolectadas 33 especies, 14 familias y 26 géneros en las cuatro estaciones. Se capturaron 984 ejemplares para una biomasa de $147410.9 \mathrm{~g}$. La mayoría de las especies son carnívoras (48\%), 33.3\% son omnívoras y $12 \%$ detritívoras y sólo dos especies son herbívoras. La especie con mayor abundancia relativa $(56.5 \%)$ y biomasa (53.7) fue la machaca (Brycon behreae). Aunque la lista de especies de peces en el río Térraba parece razonablemente completa, el conocimiento sobre los patrones ictiogegráficos es superficial. El principal componente comunitario son los peces de agua dulce secundarios con 17 especies estuarinas y una especie introducida (tilapia $O$. niloticus). Nueve especies son registradas por primera vez. El índice de diversidad $\mathrm{H}^{\prime}$ varió de 2.32 (El Brujo) a 1.67 (Coto), un patrón similar se encontró para los otros índices aplicados. Muchos de nuestros resultados fueron similares a los de previos estudios sobre distribución de peces de agua dulce en otras zonas, sin embargo no se encontró correlación significativa entre la distribución de especies y las variables ambientales. Se hipotetiza que la profundidad, velocidad del agua y atributos geomorfológicos son las variables físicas que influencian la distribución de peces. Los resultados de este estudio difieren de la tendencia de que la riqueza de especies se incrementa desde la parte alta hacia la boca del río, esta condición se atribuye a dos actividades humanas (descarga al canal principal de desechos de los cultivos de piña y sedimentos contaminados). Estas actividades podrían ser, en el futuro, una amenaza real sobre los peces y otros organismos acuáticos. Se deben estudiar otros aspectos de la historia natural de los peces de la zona, tales como crecimiento, competencia, depredación, organización comunitaria y los factores físicos y/o bióticos relacionados con la distribución ictiofaunística.

Palabras clave: Peces de agua dulce, riqueza de especies, abundancia, distribución espacial y temporal, río Grande de Térraba, Costa Rica.

\section{BIBLIOGRAFIA}

Allan J. 1995. Stream Ecology. Structure and function of running waters. Michigan. EEUU.

Alpírez, O. 1985. Ictiofauna de la vertiente Pacífica de Costa Rica. Brenesia 24: 297-318.

Angermeier, P. \& M. Winston 1988. Local vs. regional influences on local diversity in stream fish communities of Virginia. Ecology 79: 911-927

Bojsen, B. \& R. Barriga 2002. Effects of deforestation on fish community structure in Ecuadorian Amazon streams. Fresh. Biol. 47: 2246-60

Bravo-Núñez, E. \& A. Yánez-Arancibia. 1979. Ecología en la boca de Puerto Real, Laguna de Términos. I. Descripción del área y análisis estructural de las comunidades de peces. An. Centro Cienc. Mar \& Limnol. Univ. Nal. Auton. México 6: 125-182.

Brigss, J. 1984. Freshwater fishes and biogeography of Central America and Antilles. Syst. Zool. 33: 428435 .

Burcham, J. 1988. Fish communities and environmental characteristics of two lowland streams in Costa Rica. Rev. Biol. Trop. 36: 273-285. 
Bussing, W. 1966. New species and new records of Costa Rican freshwater fishes with a tentative list of species. Rev. Biol. Trop. 14: 205-249.

Bussing, W. 1974a. Pterobrycon myrnae, a remarkable new glandulocaudine characid fish from Costa Rica. Rev. Biol. Trop. 22: 135-159.

Bussing, W. 1974b. Two new species of cichlid fishes, Cichlasoma sajica and $C$. diquis, from southeastern Costa Rica. Rev. Biol. Trop. 22: 29-49.

Bussing, W. 1976. Geographical distribution of the San Juan ichthyofauna of Central America, with remarks in its origin and ecology, p. 157-175. In. T. B. Thorson (ed.), Investigations into the ichthyofauna of Nicaraguan lakes. Nebraska, EEUU.

Bussing, W. 1980. Status of the cyprinodontid fish genus Rivulus in Costa Rica, with descriptions of new endemic species. Brenesia 17: 327-364.

Bussing, W. 1985a. Roeboides ilseae, a new scale-eating characid fish from Costa Rica. Rev. Biol. Trop. 33: $45-50$.

Bussing, W. 1985b. Patterns of distribution of the Central American Ictiofauna, p. 453-473. In: S. Francis \& D. Webb (eds). The Great American Biotic Interchange. Plenum, Nueva York, EEUU.

Bussing, W. 1998. Peces de las aguas continentales de Costa Rica. Editorial Universidad de Costa Rica, San José, Costa Rica.

Bussing, W. \& M. López. 1977. Distribución y aspectos ecológicos de los peces de las cuencas hidrográficas de Arenal, Bebedero y Tempisque, Costa Rica. Rev. Biol. Trop. 25: 13-37.

Bussing, W., L. McDade \& K. Bawa. 1994. Aspectos ecológicos de la comunidad de peces. La Selva, p. 195-198. In L. McDade, K. Bawa, H. Hespenheide \& G. Harston (eds.). La Selva: ecology and natural history of a tropical rain forest. University of Chicago, Illinois, EEUU.

Camacho, R. 1967. Expedición de Gil González Dávila a Costa Rica. Tesis de Licenciatura. Universidad de Costa Rica, San José, Costa Rica.

Chicas, F. 2001. Peces juveniles en una poza provocada por la marea, Reserva Forestal Térraba-Sierpe, Puntarenas, Costa Rica. Rev. Biol. Trop. 49: 307314.

Conover, W. 1971. Practical nonparametric statistics. Nueva York, EEUU.
Constantz, G., W. Bussing \& W. Saul. 1981. Freshwater fishes of Corcovado National Park, Costa Rica. Proc. Acad. Nat. Sci. Philad. 133: 15-19.

Corrales, F., I. Quintanilla y O. Barrantes. 1988. Historia precolombina y de los siglos XVI y XVII del sureste de Costa Rica. San José. San José, Costa Rica.

Cruz, G. 1987. Reproductive biology and feeding habitats of cuyamel, Joturus pichardi, and tepemechín, Agonostomus monticola (Pisces: Mugilidae) from Río Plátano, Mosquitia, Honduras. Bull. Mar. Sci. 40: 63-72.

Estado de la Nación 2006. Décimo-segundo informe del Programa Estado de la Nación/CONARE/Defensoría de los habitantes, Costa Rica.

Fishbase 2006. Conceptos, estructura y fuentes de datos. ICLARM, Manila, Filipinas.

Gilliam, J., D. Fraser, and M. Alkins-Koo. 1993. Structure of a tropical fish community: a role for biotic interactions. Ecology 74: 1856-1870.

Henderson, P. \& R. Seaby. 1998. Species diversity and richness (also available on-line: http//www.irchouse. demon.co.uk/).

Honran, D., J. Kershner, C. Hawkings \& T. Crowl. 2000. Effects of habitat area and complexity on Colorado River cutthroat trout density in Uinta Mountain Stream. Trans. Amer. Fish. Soc. 129: 1250-1263.

ICE. 1999. Climatología y sedimentos de la cuenca río Grande de Térraba. Informe técnico. Instituto Costarricense de Electricidad. Costa Rica.

Jackson, D. \& H. Harvey. 1989. Biogeographic associations in fish assemblages: local vs. regional process. Ecology 70: 1472-1484.

Linares, O. \& A. Ranere. 1980. Adaptative radiations in Prehistoric Panama, Harvard, Cambridge, EEUU. Lyons, J. \& D. Schneider. 1990. Factors influencing fish distribution and community structure in a small coastal river in southwestern Costa Rica. Hydrobiologia 203: 1-14.

Meek, S. 1914. An annotated list of fishes known to occur in the fresh-waters of Costa Rica. Field Mus. Nat. Hist. Publ. Zool. 10: 101-134.

Meffe, W. \& L. Minckley 1987. Persistence and Stability of Fish and Invertebrate Assemblages in a Repeatedly Disturbed Sonoran Desert Stream. Amer. Mid. Natur. 117: 177-191. 
Menge, B. \& A. Olson. 1990. Role of scale and environmental factors in regulation of community structure. Trends in Ecology and Evolution 5: 52-57.

Miller, R. 1966. Geographical distribution of Central America freshwater fishes. Copeia 4: 773-802.

Myers, G. 1966. Derivation of the freshwater fish fauna of Central America. Copeia 1966: 766-773.

Oro, G. \& P. Cabrera. 1993. La tilapia Oreochromis niloticus (Pisces: Cichlidae) en el Caribe norte de Costa Rica. Rev. Biol. Trop. 41: 920-921.

Pizarro F. \& J. Rojas. 1993. Presencia de Oreochromis niloticus en la desembocadura del río Bebedero, Golfo de Nicoya, Costa Rica. Rev. Biol. Trop. 41: 921-924.

Regan, C. 1907. Descriptions of six new freshwater fishes from Mexico and Central America. Ann. Mag. Nat. Hist. 7: 258-260.

Regan, C. 1908. A collection of freshwater fishes made by Mr. C. F. Underwood in Costa Rica. Ann. Mag. Nat. Hist. 8: 455-464.

Ricklefs, R. E. 1987. Community diversity: relative roles of local and regional processes. Science 235: $167-$ 171.

Ricklefs, R. \& D. Schluter. 1993. Species diversity: regional and historical influences, p. 350-363. In R. Ricklefs \& D. Schluter (eds.). Species diversity in ecological communities: historical and geographical perspectives. University of Chicago, Chicago, EEUU.

Rojas, J. \& O. Rodríguez. 2005. Los peces y los estudios de viabilidad ambiental de proyectos hidroeléctricos: $\mathrm{PH}$ Boruca un caso de estudio. Energía 45: 22-26.

Rojas, J. \& O. Rodríguez. 2006. Peces y viabilidad ambiental. VIDA 1: 14-16.

Rojas, J., F. Pizarro \& M. Castro. 1994. Diversidad y abundancia íctica en tres áreas de manglar en el Golfo de Nicoya, Costa Rica. Rev. Biol. Trop. 42: 663-672.
Roldan, G. 1992. Fundamentos de limnología neotropical. Antioquia. Medellín, Colombia.

Sáenz, I., M. Protti \& J. Cabrera. 2006. Composición de especies y diversidad de peces en un cuerpo de agua temporal en el Refugio Nacional de Vida Silvestre Caño Negro, Costa Rica. Rev. Biol. Trop. 54: 639645.

Schiemer F. 2000. Fish as indicators for the assessment of the ecological integrity of large rivers. Hydrobiologia 422/423: 271-278

Schlosser, I. 1991. Stream fish ecology: a landscape perspective. Bioscience 41: 704-712.

UICN-ORMA, 1995. El Humedal de Sierpe-Térraba. UICN, San José, Costa Rica. 56 p.

Vannote, R., G. Minshall, K. Cummings, J. Seddel, \& C. Cushing. 1980. The river continuum concept. Can. J. Fish. Aquat. Sci. 37: 130-137

Winemiller, K. 1983. An introduction to the freshwater fish communities of Corcovado National Park, Costa Rica. Brenesia 21: 47-66.

Winemiller, K. 1993. Seasonality of reproduction by livebearing fishes in tropical rainforest streams. Oecologia 95: 266-276.

Winemiller, K. \& N. Morales. 1989. Comunidades de peces del Parque Nacional Corcovado luego del cese de las actividades mineras. Brenesia 31: 75-91.

Wootton, J. \& M. Oemke. 1992. Latitudinal differences in fish community trophic structure, and the role of fish herbivory in a Costa Rican stream. Envir. Biol. Fish. 35: 311-319.

Yánez-Arancibia, A., F. Amescua-Linares \& J. Day. 1980. Fish community structure and function in Términos Lagoon. A tropical estuary in the southern Gulf of Mexico, p. 465-482. In V. Kennedy (ed). Estuarine Perspectives. Academic. Nueva York, EEUU. 
